\title{
The genetic landscape of hepatitis delta virus infection
}

\author{
Authors: Carola Maria Bigogno, ${ }^{\mathrm{A}}$ Roya Razavi Falahieh, ${ }^{\mathrm{A}}$ Kai Man Alexander Ho, ${ }^{\mathrm{A}}$ Anna Jeffrey-Smith, ${ }^{\mathrm{A}}$ \\ Meleri Jones, ${ }^{\mathrm{A}}$ Carla Usai, ${ }^{\mathrm{A}}$ Arianna Battisti, ${ }^{\mathrm{A}}$ Upkar Gill ${ }^{\mathrm{A}}$ and Patrick Kennedy ${ }^{\mathrm{A}}$
}

\section{Introduction}

Hepatitis delta virus (HDV) is a small, defective ribonucleic acid (RNA) virus that requires hepatitis B virus (HBV) for entry into hepatocytes, but its genome replication is independent of HBV. Over 15 million people are infected by HDV worldwide, always occurring in the presence of HBV. ${ }^{1} \mathrm{HBV} / \mathrm{HDV}$ coinfection is associated with an acceleration of liver disease, leading to cirrhosis, liver cancer and eventually liver failure. Transmission occurs parenterally through contact with infected blood and body fluids. ${ }^{2}$

HDV is highly variable, and eight genotypes have been identified to date. ${ }^{3}$ This variability has a great impact on identifying standardised diagnostic tools, as well as specific direct anti-HDV drugs. Currently, the standard treatment for HDV consists of a 48-week course of pegylated interferon $\alpha$ (PEG-IFN- $\alpha$ ), but it is highly ineffective, with a sustained virological response rate of $25 \% .{ }^{4}$ Novel agents such as Myrcludex B have shown promise but remain in the development pipeline.

The aim of the study is to sequence and genotype HDV samples of patients managed at the Royal London Liver Unit. Moreover, mutational analysis was conducted to confirm the high variability of HDV.

\section{Materials and methods}

Sixteen patients with HDV were identified from the Liver Unit at Barts Health NHS Trust. Ten (62.5\%) were male, with a median age of 37 years old. Ten $(62.5 \%)$ patients were originally from Europe, four (25\%) from Africa and two (12.5\%) from Asia.

Plasma samples were collected and viral RNA was inactivated and extracted. Reverse transcription was performed to obtain viral complementary deoxyribonucleic acid. A specific sequence of the virus was amplified, which was then sequenced using the Sanger technique. Mutational analysis and sequence alignment allowed the generation of a phylogenetic tree using $\mathrm{MEGA}^{\circledR}$.

\section{Results and discussion}

Mutational analysis showed the presence of quasispecies, which define the high variability of nucleotides within the viral pool in each patient. This is due to the high replication rate of the virus and the lack of an error-checking mechanism. Sequence alignment also confirmed the high variability of HDV.

Finally, phylogenetic analysis using $\mathrm{MEGA}^{\odot}$ allowed genotyping and subtyping of the 16 samples. Fifteen samples were found to be genotype 1 . This was coherent with our expectations, considering that genotype 1 is the most prevalent. One sample was found to be genotype 5 - this was also coherent, as genotype 5 is predominantly found in sub-Saharan Africa, which is where this specific patient is originally from.

\section{Conclusion}

In conclusion, the project aims were met, and the study provides valuable information which will add to the current limited understanding of HDV.

Future work will focus on full-length sequencing and nextgeneration analysis of HDV from the same cohort of patients. This will aid in the identification of the HDV quasispecies, which will be used to provide a better understanding of the biological events in HDV infection, as well as to improve diagnostic assays and treatment decisions.

\section{Conflicts of interest}

None declared.

\section{References}

1 Taylor JM. Hepatitis D virus replication. Cold Spring Harb Perspect Med 2015;5:a021568.

2 Farci P. Delta hepatitis: an update. J Hepatol 2003;39 Suppl 1:S2129.

3 Hughes SA, Wedemeyer H, Harrison PM. Hepatitis delta virus. Lancet 2011;378:73-85.

4 Farci P, Mandas A, Coiana A et al. Treatment of chronic hepatitis D with interferon alfa-2a. N Engl J Med 1994;330:88-94. 